\title{
MRI ROLE IN CHEMOTHERAPEUTIC INDUCED PRES
}

\section{Samar Ramzy Ragheb ${ }^{*}$ and Sherine Mohamed Sharara ${ }^{*}$}

\author{
Department of radiodiagnosis, \\ Faculty of medicine, Ain Shams \\ university, Cairo, Egypt \\ Corresponding : \\ Samar Ramzy Ragheb \\ Mobile: 01003568254 \\ E mail: \\ doc mina2004@yahoo.com \\ Received: 4/12/2019 \\ Accepted: $28 / 12 / 2019$
}

Online ISSN: 2735-3540

\begin{abstract}
:
Background: Posterior reversible encephalopathy syndrome is a very common neurotoxic side effect of chemotherapeutics.

Aim of the work: To display the importance of Magnetic resonance imaging in early detection of the chemotherapeutic induced neurotoxicity.

Patients \& Methods: Fifty cancer patients (12 females and 38 males), their age ranged from 2 to 61 years on chemotherapy having neurological manifestations. Magnetic resonance imaging was performed to evaluate the radiological findings of neurological manifestations.
\end{abstract}

Results: 31 cases were found to have Posterior reversible encephalopathy syndrome, while the others 19 cases showed other complications.

Conclusion: MRI showed an important role in detecting PRES in cancer patients due to chemotherapeutics.

Key words: MRI- Chemotherapeutics-PRES

\section{INTRODUCTION:}

The chemotherapeutic agents became widely used especially in cancers although their side effects are unfortunately common. Differentiation between the pathology of a disease as cancer and the side effects of chemotherapeutics is still representing a challenge ${ }^{(1 \& 2)}$.

Chemotherapeutics can cause CNS neurotoxicity besides peripheral neurotoxicity despite the protective role of BBB. And for that reason the dose of chemotherapy in many patients is started to be limited. The development of the neurotoxic effects of chemotherapeutics may be acute, sub acute, or delayed, and may be reversible or partially irreversible ${ }^{(3 \& 4)}$.

These effects can be presented at the same time or may occur as isolated symptoms. For example; seizures, aphasia, hemi paresis, and cortical blindness ${ }^{(5)}$.

A very common neurotoxic side effect of chemotherapeutics is Posterior reversible encephalopathy syndrome [PRES] in which there is cortical and sub cortical symmetrical vasogenic edema within the parietal and occipital regions in most cases. Although it is termed posterior, PRES can be found in areas other than posterior distribution for example; frontal, inferior temporal, cerebellar and brainstem regions ${ }^{(6-9)}$.

The management plan of neurotoxicity caused by chemotherapeutics remains supportive after excluding infectious causes $^{(10)}$.

\section{AIM OF THE WORK:}

To display the importance of Magnetic resonance imaging in early detection of the chemotherapeutic induced neurotoxicity.

\section{PATIENTS \& METHODS:}

The current study included fifty cases of different cancers with CNS radiological 


\section{Samar Ramzy Ragheb ${ }^{*}$ and Sherine Mohamed Sharara}

findings. These patients had received chemotherapeutics according to their primary cancer protocol. All these patients were presented to the radiology department in the National Cancer Institute (NCI) at Cairo University in the time interval between 2015 and 2017.

\section{Inclusion criteria:}

1. Any cancer patient.

2. Any age, sex and $1^{\text {ry }}$ cancer.

3. Receiving any protocol of chemotherapy.

4. Complaining with neurological manifestations such as signs of increase ICP (headache, nausea, emesis, and blurred vision), convulsions aphasia, hemiparesis, or paresis.

\section{Exclusion criteria:}

1. Patients with absolute contraindication to MRI: Patients with Pacemaker or metallic implant.

2. Patients with contraindication to contrast: Patients with disturbed renal function test (if creatinine $>2$ ), patients with glomerular filtration rate $<30 \mathrm{ml}$ per min. per $1.73 \mathrm{~m} 2$ or any acute renal insufficiency related to the hepato-renal syndrome or perioperative liver transplantation.

3. Patients with primary CNS tumor or metastatic brain tumor.

\section{Patient preparation:}

Detachable metallic implants like teeth prosthesis are considered as proportional contraindication should be removed prior to entrance to magnetic area. For the patient needed anaesthesia, fasting 4-6 hours before the scan is required.

\section{MRI technique}

\section{Conventional contrast enhanced MRI (CEMRI):}

All patients underwent the CEMRI on 1.5 T Philips Achieva scanner MRI system using a 16-channel sensitivity-encoding (SENSE) head coil

All cases were examined using the following protocol:

- Axial T1-weighted images: (TE=15 $\mathrm{m} / \mathrm{sec} \mathrm{TR}=581 \mathrm{~m} / \mathrm{sec}$ )

- Axial fast spin-echo T2-weighted images:(TE=110 m/sec TR=4846 m/sec)

- Axial and sagittal Fluid-Attenuated Inversion- Recovery sequences (FLAIR): $(\mathrm{TE}=140 \quad \mathrm{~m} / \mathrm{sec}, \quad \mathrm{TR} / \mathrm{TI}=10000 / 2800$ $\mathrm{m} / \mathrm{sec}$ ).

After IV administration of Gd- DTPA (o. $3 \mathrm{mg} / \mathrm{kg}$ ), contrast enhanced T1WI in three orthogonal planes are obtained.

\section{Diffusion weighted MR imaging:}

Single shot spin echo EPI sequence (TR/TE/NEX: 3430/108 ms/I) with diffusion sensitivities of $b$ values $=0$ and $1000 \mathrm{~s} / \mathrm{mm} 2$.

$\mathrm{FOV}=22 \mathrm{~cm}$ in axial images and $25 \mathrm{~cm}$ in sagittal images.

- Matrix (frequency x phase) 220x140

- Slice thickness $=5 \mathrm{~mm}$ with $1 \mathrm{~mm}$ interval. (In all sequences)

The ADC maps were calculated automatically by MRI software and included in the sequence.

\section{Data collection:}

Data collected include the following:

- ID of patients, age at diagnosis \& gender.

- Primary cancer.

- The type of chemotherapy given $\&$ the route of administration.

- Clinical picture \& the neurological presenting symptom.

- Provisional diagnosis and assessment.

We analyzed the results of cranial neuroimaging studies in all patients referred to our department for investigation of neurologic symptoms and signs. The medical records were reviewed with attention especially to the type of chemotherapy 
given, the onset of symptoms, and the CNS complications.

\section{Image Interpretation:}

For the sake of better characterization of the CNS changes, all the available images were assessed.

Regarding PRES syndrome, the images were assessed for determination of the disease bilaterality, symmetricity, distribution of specific lobe \& the presence of any basal ganglia or infra-tentorial areas involvement (cerebellum \& brain stem). Images are also reviewed in conjunction with the diffusion images providing a better characterization of the type of edema present whether cytotoxic or vasogenic.

\section{RESULTS:}

\section{Demographic Data}

The pool of this study is 50 cancer patients (Table 1) on Chemotherapy (38 male and 12 female) having CNS manifestations. Their ages ranged from 2 to 61 years. MRI was performed to evaluate the radiological findings of CNS manifestations. These manifestations have developed $2^{\text {ry }}$ to the chemotherapeutic drugs given to the patient according to the protocol of treatment.

Table 1. Demographic data of the studied patients $(\mathrm{No}=50)$.

\begin{tabular}{|c|c|c|}
\hline Demographic Data & \multicolumn{2}{|c|}{ All studied patients $(\mathrm{n}=50)$} \\
\hline Gender & No. & $\%$ \\
\hline Female & 12 & $76 \%$ \\
\hline Male & 38 & \\
\hline Age & \multicolumn{2}{|c|}{$15 \pm 14$} \\
\hline Mean \pm SD & \multicolumn{2}{|c|}{$11(2-61)$} \\
\hline Median (Range) &
\end{tabular}

The patients' age are divided into:

- Pediatric $(<18$ year old) $(44 / 50)$

- $\quad$ Adult (>18 year old) $(6 / 50)$

The primary cancer of the patients is demonstrated in (Fig. 1). It showed that hematological cancers were the commonest $1^{\text {ry }}$ cancers among all patients representing more than $85 \%$ of the cases in which ALL
$(54 \%)(\mathrm{N}=27)$ was the most common cancer among them, followed by NHL (36\%) $(\mathrm{N}=18)$. Then mucinous colonic adenocarcinoma $(6 \%)(\mathrm{N}=3)$ \& cancer pancreas $(4 \%)(\mathrm{N}=2)$

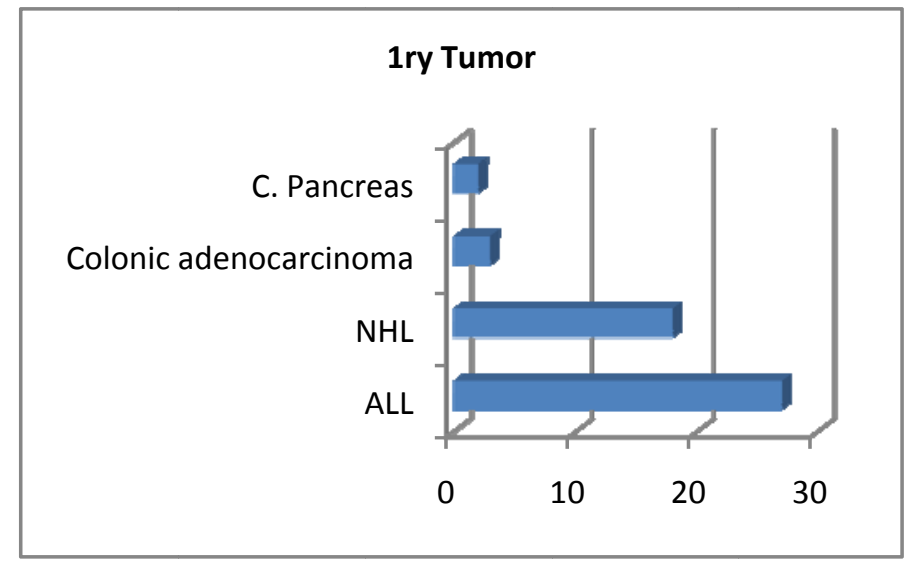

Fig.1 Bar chart shows primary tumor of the patients. 


\section{Samar Ramzy Ragheb ${ }^{*}$ and Sherine Mohamed Sharara}

\section{Clinical Data}

In current study, the commonest presentation (Table 2) was severe persistent headache that present in $(70 \%)$ (35 out of 50 ), followed by seizures, that present in
$(20 \%)$ (10 out of 50$)$. Also, there were another presenting symptoms like; visual deficit $(6 \%)$ (3 out of 50$)$, altered mental status and facial palsy (just one case for each) ( $2 \%$ each).

Table 2. Clinical presentation of the patients

\begin{tabular}{|c|c|c|}
\hline Symptom & No. & $\%$ \\
\hline Headache & 35 & $70 \%$ \\
\hline Seizures & 10 & $20 \%$ \\
\hline Visual deficit & 3 & $6 \%$ \\
\hline Altered mental status & 1 & $2 \%$ \\
\hline Facial palsy & 1 & $2 \%$ \\
\hline
\end{tabular}

\section{Types of chemotherapeutic neurotoxicity:}

PRES (31 out of 50) was the most common CNS abnormality found (Fig. 2), while the others 19 cases showed other complications e.g. sinus thrombosis.

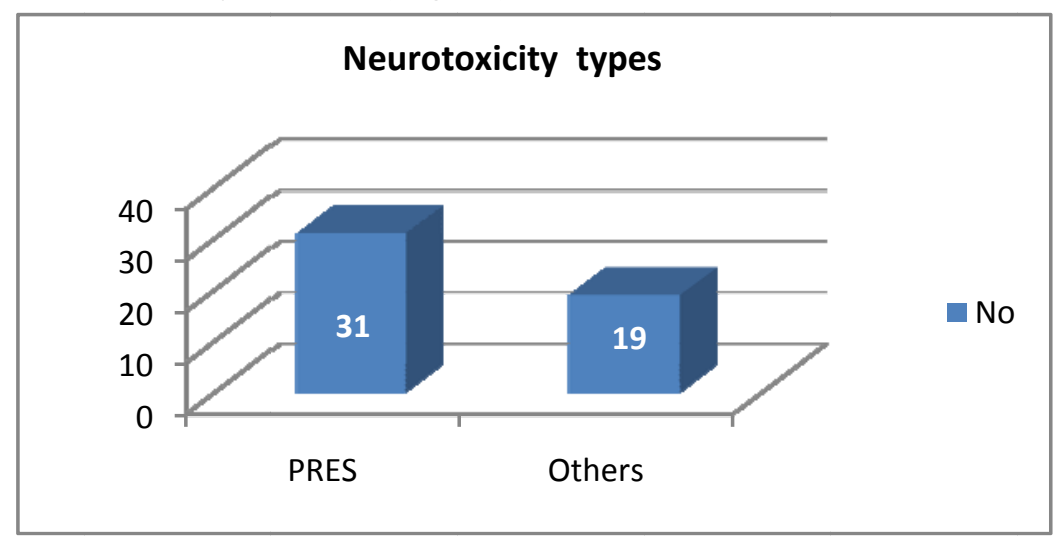

Fig. 2 Chart shows types of chemotherapy induced neurotoxicity.

\section{Initial MRI:}

All patients underwent MRI with time interval between the clinical onset and date of MRI demonstrated in (Table 3), in which the lowest interval is of the patients underwent MRI in the same day of clinical onset and the longest duration is 10 days after onset of the disease.

Table 3. Time interval between onset of the disease and initial MRI

\begin{tabular}{|c|c|}
\hline Time interval & (in days) \\
\hline Mean & 1.5 \\
\hline Median & 0.5 \\
\hline Standard deviation & 2.5 \\
\hline Minimum & 0 \\
\hline Maximum & 10 \\
\hline
\end{tabular}

\section{Posterior Reversible Encephalopathy Syndrome:}

31 patients out of 50 patients had developed PRES. They are classified as: 27 pediatric $(<18$ years old) and 4 adult $(>18$ years old), 23 males and 8 females. The lesions demonstrated hyper intense signal on T2WI and FLAIR images and iso/hypointense signal T1WI in all patients.

\section{Location of PRES:}

In our patients, predominant involvement of the cerebral cortex was seen in 
$67.7 \%$ (21 out of 31), subcortical predominant involvement was seen in $19.35 \%$ (6 out of 31) and involvement of both of them was seen in $12.9 \%$ ( 4 out of 31 ) of the patient as demonstrated in (Fig. 3).

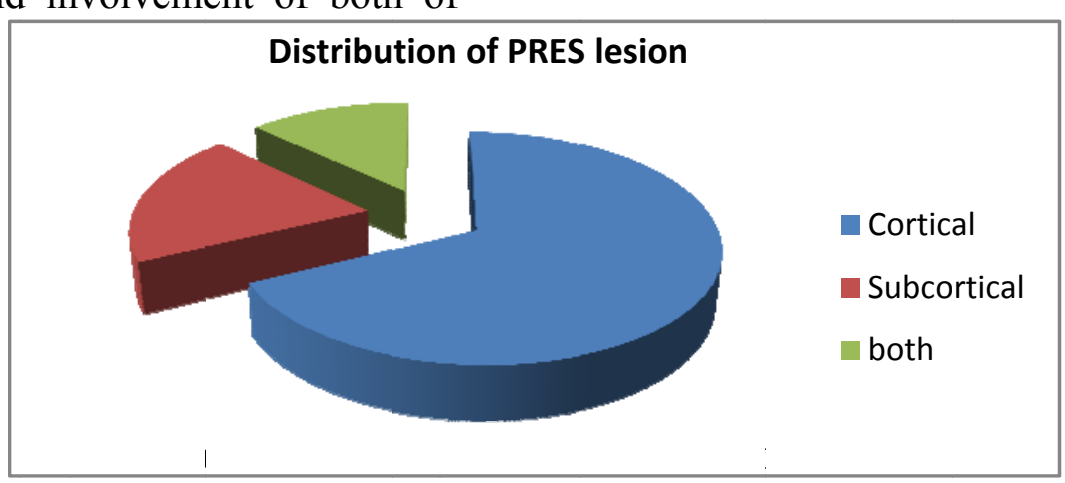

In current study, the commonest involved areas were the parieto-occipital lobes $(90.3 \%)$ (28 out of 31$)$. These areas are considered the typical distribution of PRES. The least affected regions (Fig. 4) were the cerebellum $(22.58 \%, 7$ cases out of 31$)$, frontal lobe $(19.35 \%, 6$ cases out of 31$)$, temporal lobe $(9.6 \%$, 3 cases out of 31$)$, basal ganglia, corpus callosum and brain stem (each represented 3.2\%1 case out of 31 ). These regions are considered the atypical distribution of PRES.

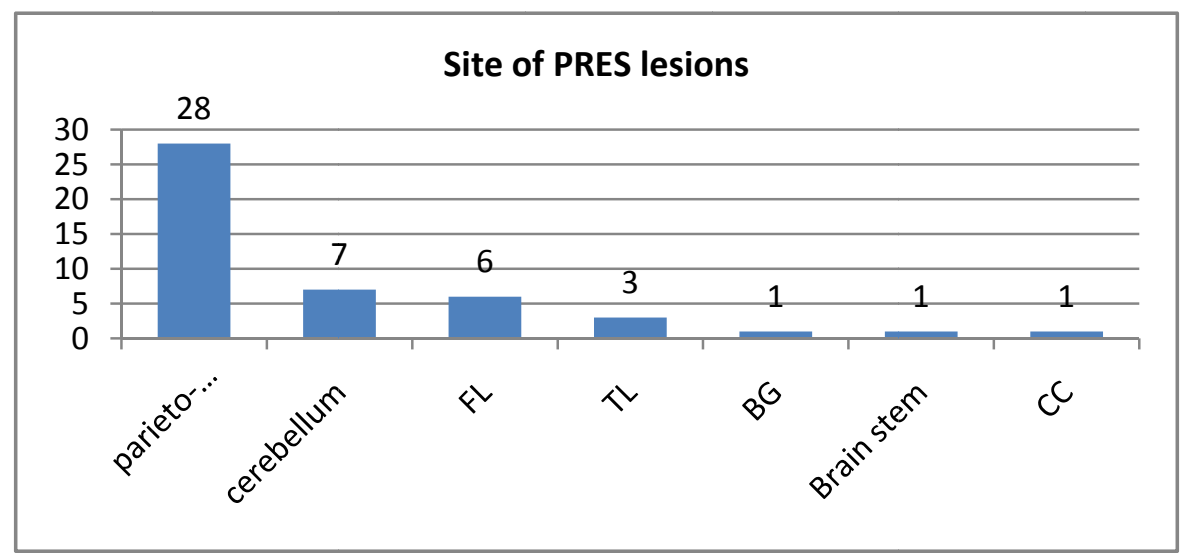

Fig. 4 Column chart showing the sites of PRES lesions

According to the division of the brain regions into three parts;

1. Anterior circulation (AC)

2. Posterior circulation (PC)

3. Deep structure (DS).

Anterior circulation includes frontal lobe, temporal lobe and parietal lobe; Posterior circulation includes occipital lobe, cerebellum and brain stem and deep structure includes basal ganglia, deep white matter and corpus callosum, $\mathrm{AC}$ regions were involved in $87 \%$ of cases and PC regions were involved in $93.5 \%$, as well as DS in $19 \%$.

Bilateral Lesions were seen in $(90.3 \%$, 28 cases out of 31$)$ and unilateral in $(9.6 \%, 3$ cases out of 31) of the patients (Fig.5). In the 28 patients with the bilateral distribution, they were re-classified as $(64.28 \%, 18$ cases out of 28$)$ were asymmetrical and $(35.7 \%$, 10 cases out of 28 ) were symmetrical. 


\section{Samar Ramzy Ragheb ${ }^{*}$ and Sherine Mohamed Sharara}

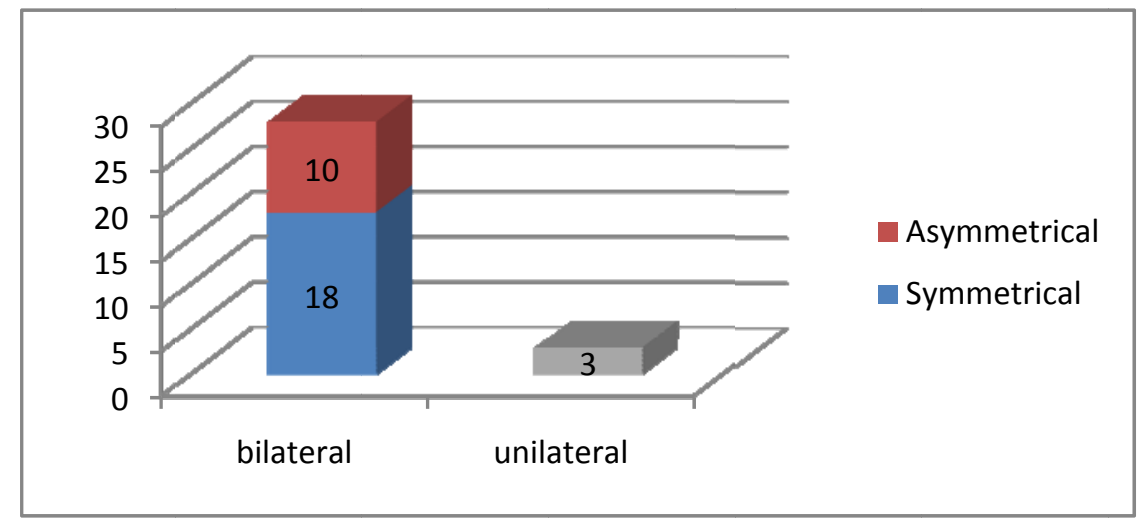

Fig. 5 Column chart showing the side of brain affected by PRES lesion.

\section{Typical versus atypical MRI findings:}

We considered the PRES to be typical if the MRI showed vasogenic edema with symmetrical distribution, mainly within the parieto-occipital regions, with no enhancement, diffusion restriction or
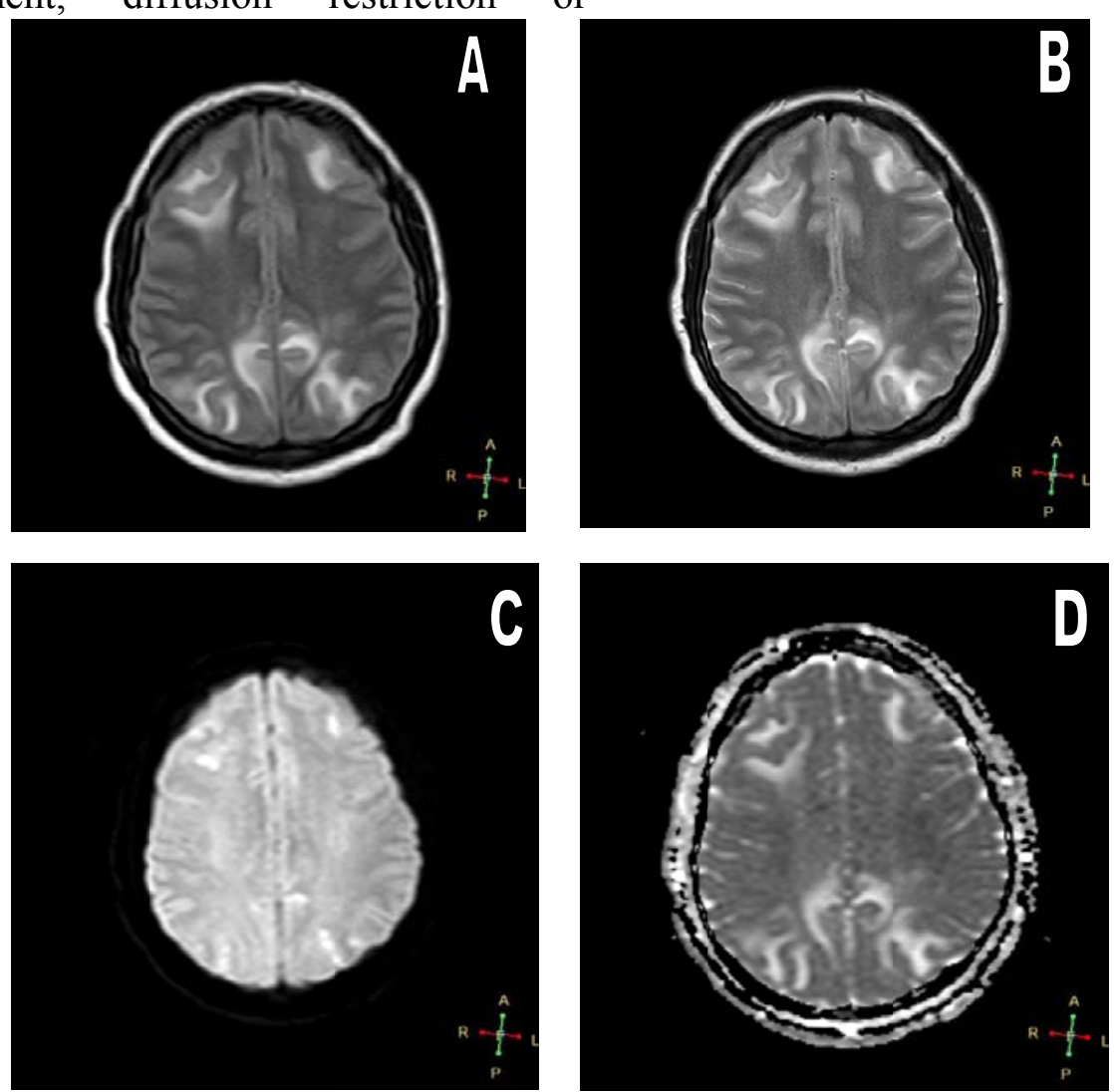

Fig. 6 25-years-old female has NHL on chemotherapy, She started to suffer from frequent fits and altered mental status, MRI was done, it showed: a) FLAIR, b) T2 show bilateral symmetrical subcortical areas of high SI in frontal and parietal regions. c) DWI, d) ADC map: show no areas of restricted diffusion. Diagnosis: MRI finding consistent of typical PRES. 

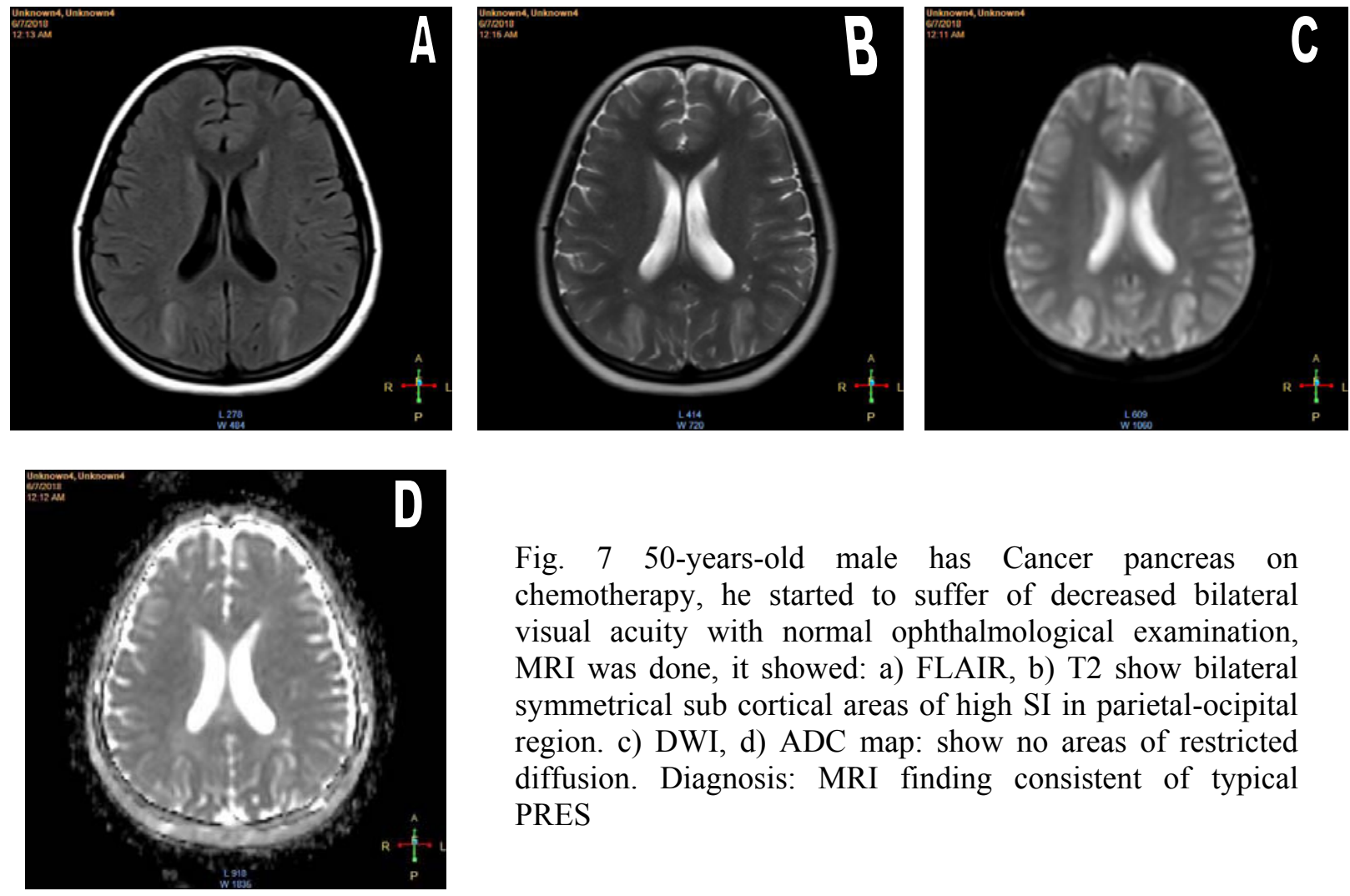

Fig. 7 50-years-old male has Cancer pancreas on chemotherapy, he started to suffer of decreased bilateral visual acuity with normal ophthalmological examination, MRI was done, it showed: a) FLAIR, b) T2 show bilateral symmetrical sub cortical areas of high SI in parietal-ocipital region. c) DWI, d) ADC map: show no areas of restricted diffusion. Diagnosis: MRI finding consistent of typical PRES
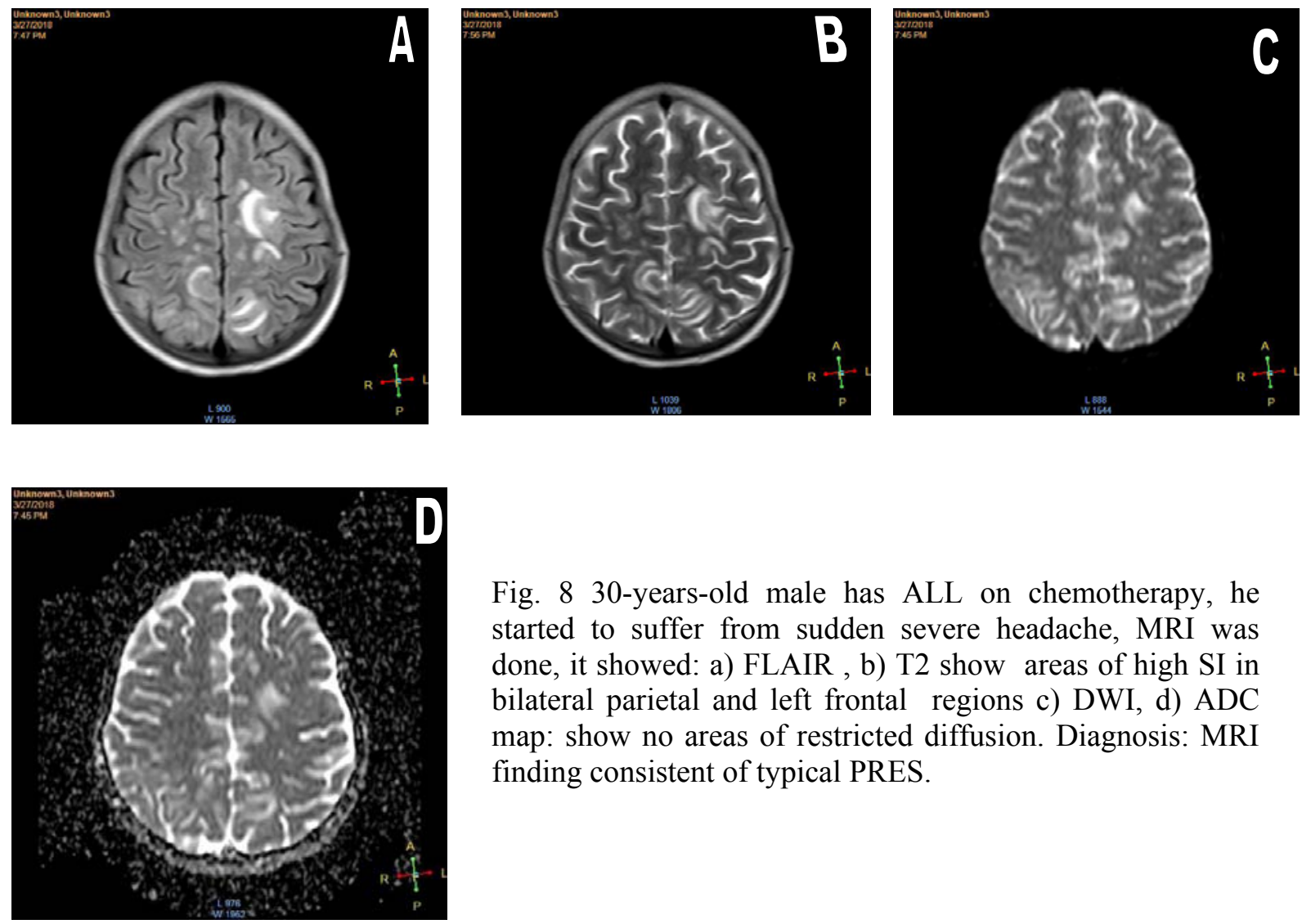

Fig. 8 30-years-old male has ALL on chemotherapy, he started to suffer from sudden severe headache, MRI was done, it showed: a) FLAIR , b) T2 show areas of high SI in bilateral parietal and left frontal regions c) DWI, d) ADC map: show no areas of restricted diffusion. Diagnosis: MRI finding consistent of typical PRES. 

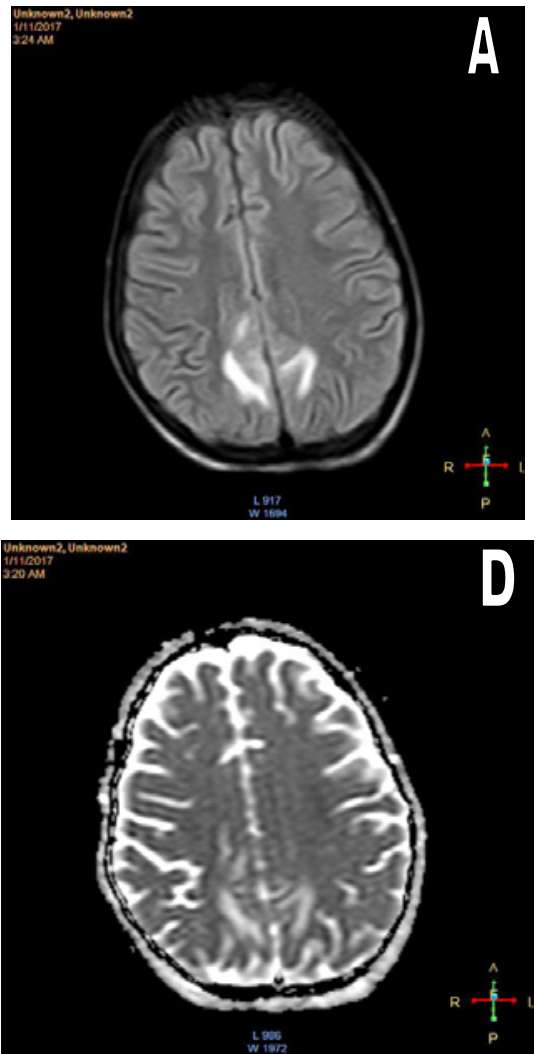

In current study, it was considered as atypical location if there is involvement of any area of the brain other than the parietooccipital regions. It represented $(66.6 \%, 14$ cases out of 21). Also, the presentation was considered to be atypical if there is asymmetrical distribution of the lesion, contrast enhancement, restricted diffusion or hemorrhage (Fig. 10, 11\&12).
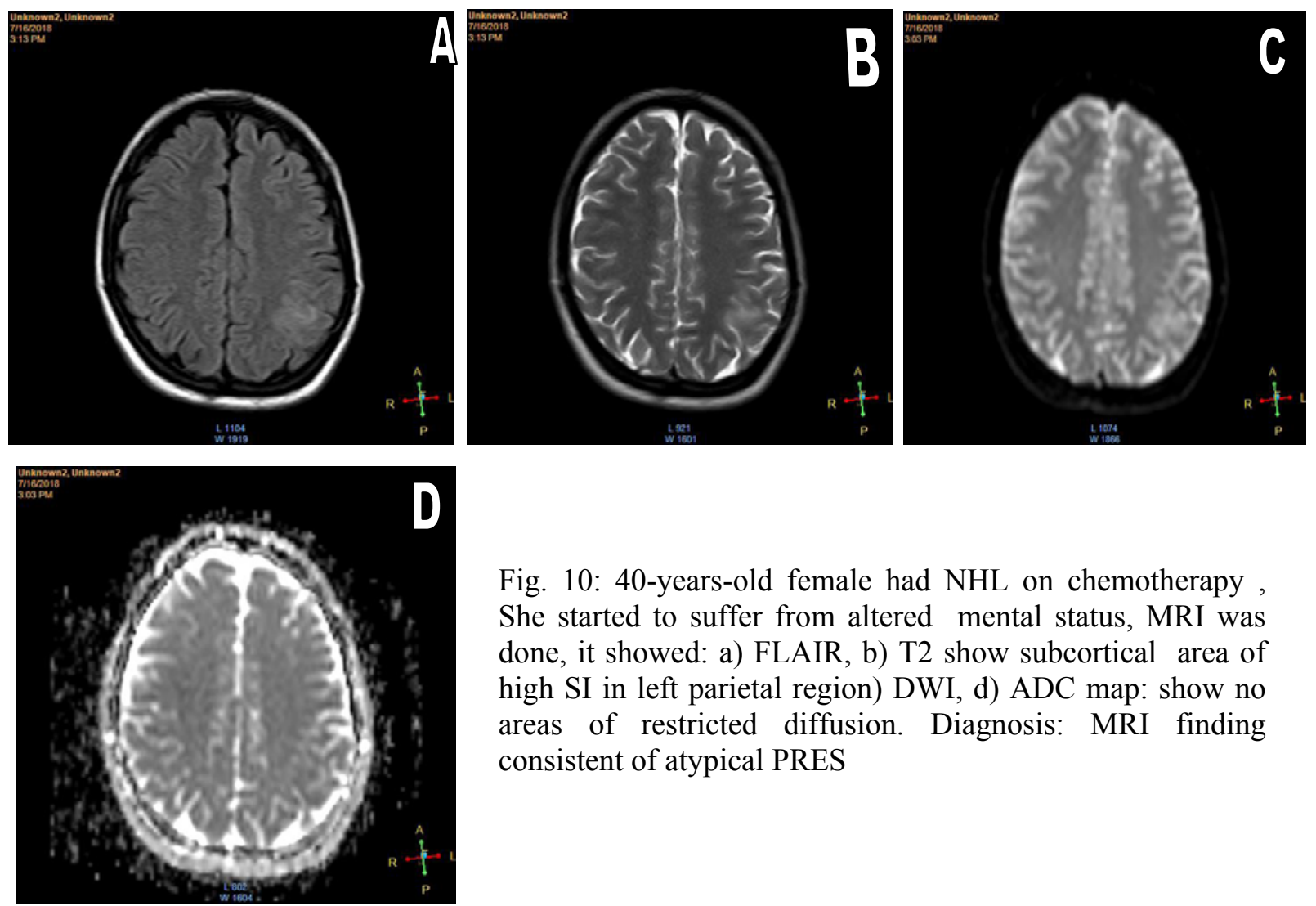

Fig. 10: 40-years-old female had NHL on chemotherapy , She started to suffer from altered mental status, MRI was done, it showed: a) FLAIR, b) T2 show subcortical area of high SI in left parietal region) DWI, d) ADC map: show no areas of restricted diffusion. Diagnosis: MRI finding consistent of atypical PRES 

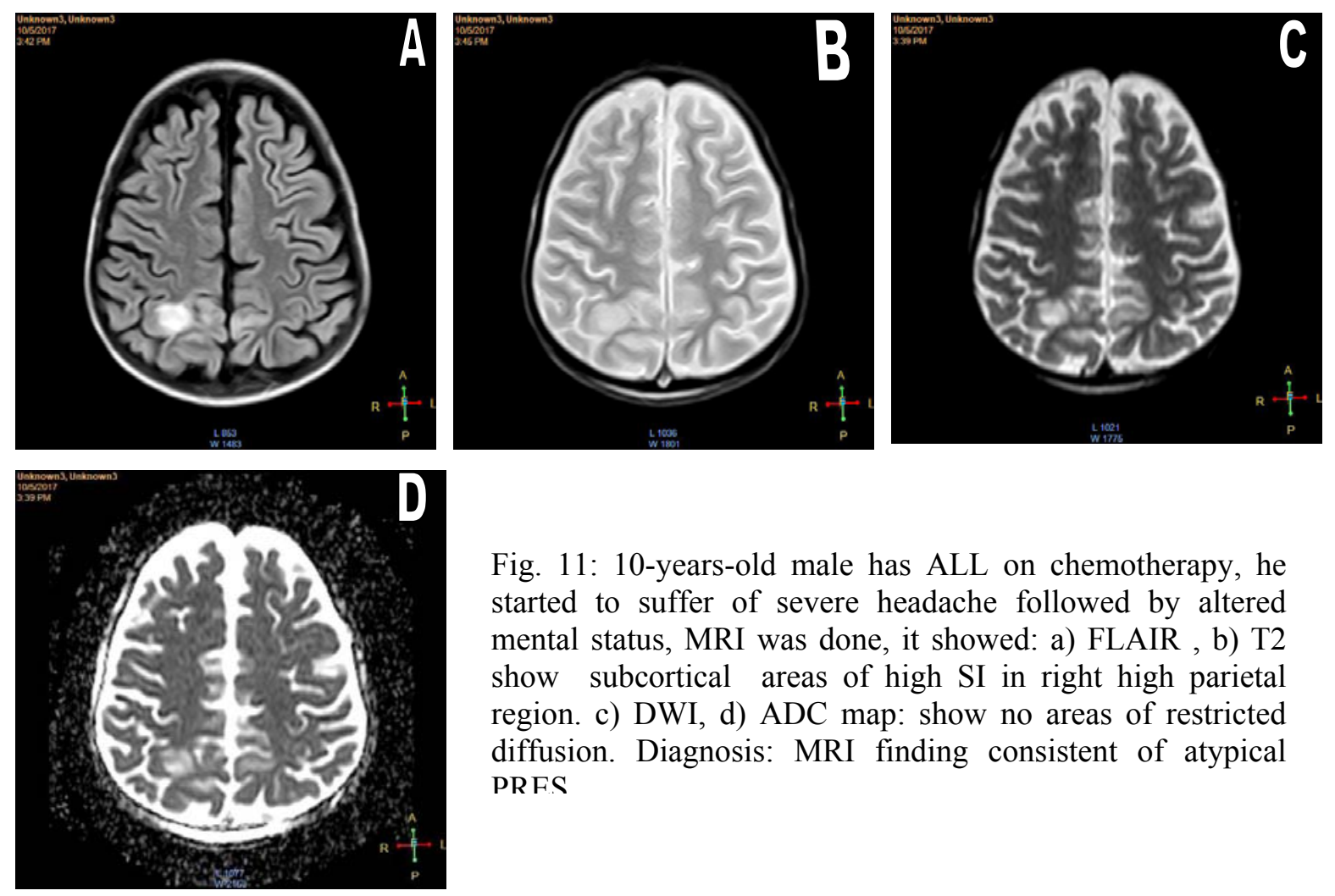

Fig. 11: 10-years-old male has ALL on chemotherapy, he started to suffer of severe headache followed by altered mental status, MRI was done, it showed: a) FLAIR , b) T2 show subcortical areas of high SI in right high parietal region. c) DWI, d) ADC map: show no areas of restricted diffusion. Diagnosis: MRI finding consistent of atypical PRFS
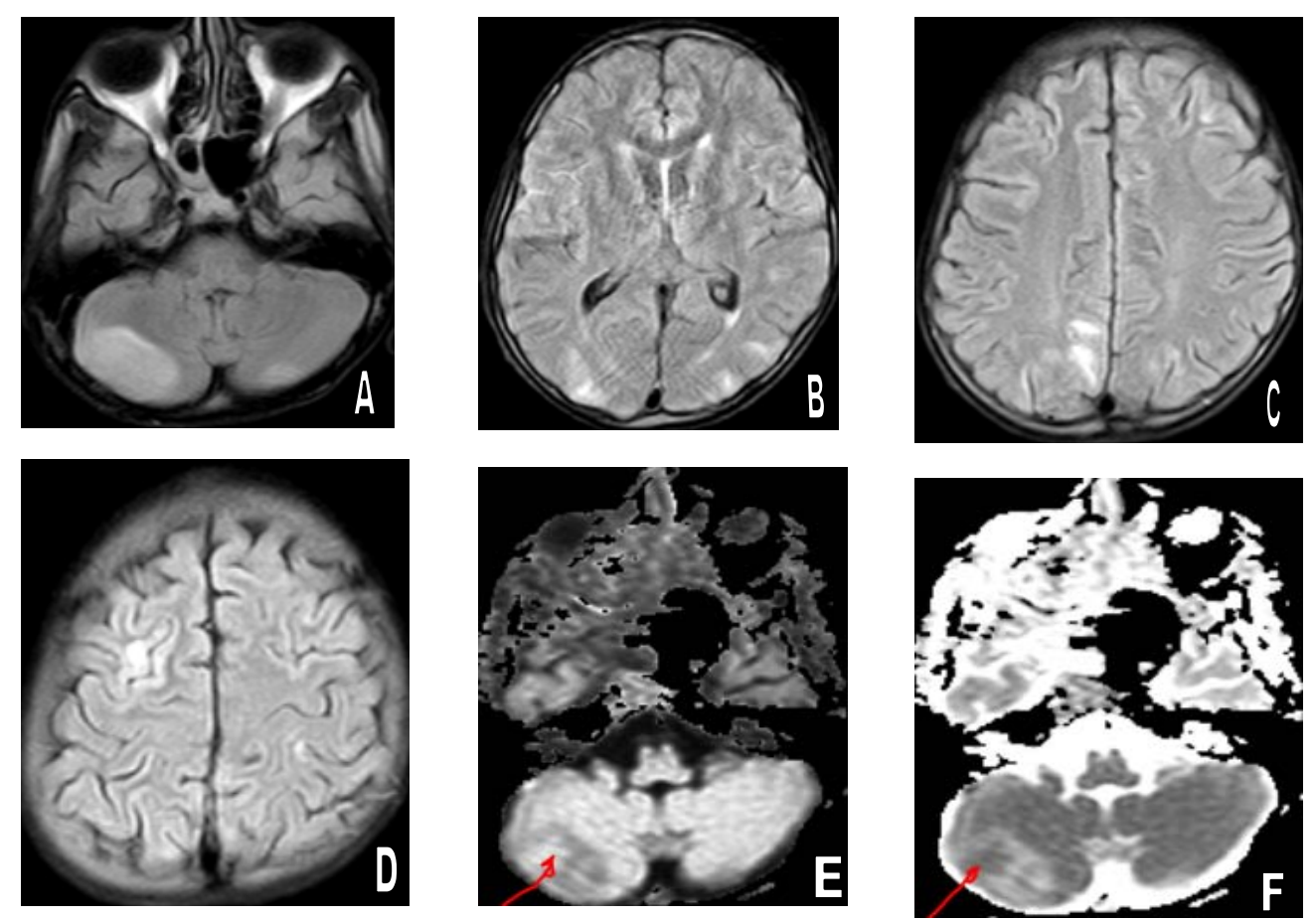

Fig. 12 13-year-old boy has NHL on cyclophosphamide, Vincristine \& Prednisolone (IV). He started to complain of generalized tonic-colonic convulsions, MRI was done immediately on the same day of his complain, it shows: A-D) FLAIR images show multiple bilateral cortical \& to lesser extent subcortical areas \& foci of high SI are noted at the bilateral cerebellar, occipital, parietal \& right frontal areas with (E) Evidence of restricted diffusion at the right cerebellar region \& low ADC map (F). Diagnosis: MRI finding impressive of atypical PRES. 


\section{Samar Ramzy Ragheb ${ }^{*}$ and Sherine Mohamed Sharara}

Also in current study, The atypical criteria of PRES (Fig.13) were asymmetrical distribution of the lesion which was found in (85.7\%, 18 cases out of 21$)$, followed by diffusion restriction which is found in
|(66.6\%, 14 cases out of 21$)$, then contrast enhancement which was seen in $(14.2 \%, 3$ cases out of 21), finally was the presence of hemorrhage (4.7\%, only one case out of 21$)$.

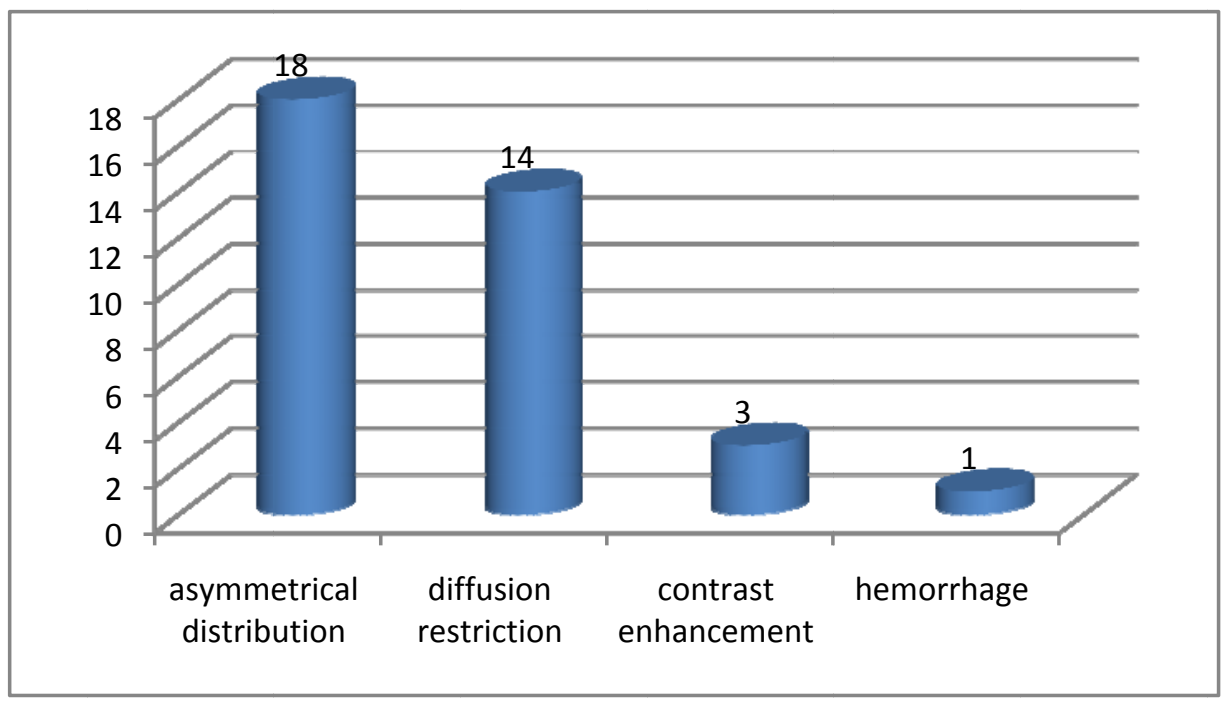

Fig. 13 Column chart showing the atypical criteria of PRES

\section{Grading of PRES:}

Grading of PRES depends on the extension of brain edema within the brain parenchyma especially on T2 /FLAIR images. The patients are divided into 4 grades (Table 4). In which:
- $\quad$ Grade 1 represented 25.8\%(8/31)

- $\quad$ Grade 2 represented 35.48\% (11/31)

- $\quad$ Grade 3 represented 12.9\% (4/31)

- $\quad$ Grade 4 represented 25.8\% (8/31)

Table 4. Grading severity of PRES

\begin{tabular}{|l|l|l|l|}
\hline \multicolumn{1}{|c|}{$\begin{array}{c}\text { Minimal } \\
\text { (Grade 1) }\end{array}$} & \multicolumn{1}{c|}{$\begin{array}{c}\text { Mild } \\
\text { (Grade 2) }\end{array}$} & $\begin{array}{c}\text { Moderate } \\
\text { (Grade 3) }\end{array}$ \\
\hline $\begin{array}{l}\text { involvement of one } \\
\text { lobe }\end{array}$ & involvement of 2 lobes & involvement of 3 lobes & $\begin{array}{l}\text { Severe } \\
\text { (Grade 4) }\end{array}$ \\
& & $\begin{array}{l}\text { involvement of all lobes from the } \\
\text { ventricular margin to the subcortical } \\
\text { white matter, or involvement of corpus } \\
\text { callosum, basal ganglia, thalami, or } \\
\text { internal capsules. }\end{array}$ \\
\hline
\end{tabular}

\section{Follow up MRI}

$(51.6 \%, 16$ cases out of 31$)$ did a follow up MRI and (48.38\%, 15 cases out of 31$)$ didn't. Complete resolution was seen in half of the patients who underwent follow up
MRI (Fig. 14) and (31.25\%, 5 cases out of 16) showed regressive course of the disease. On other hand, $(12.5 \%, 2$ cases out of 21$)$ showed progressive course (Fig. 15) and just one case $(6.25 \%)$ showed stationary course. 

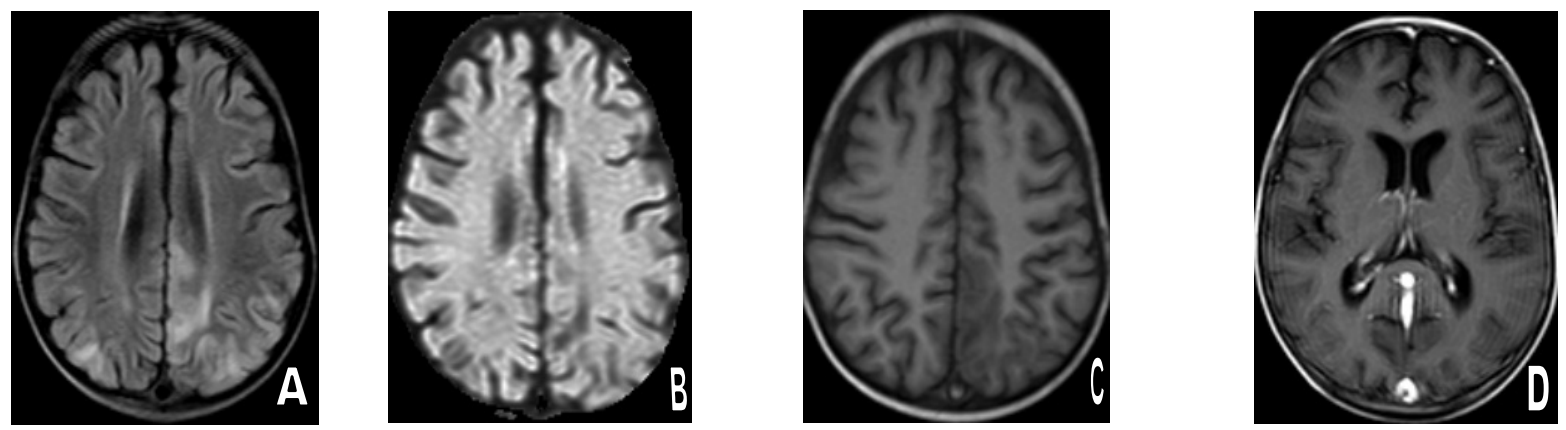

Fig.14.1 3-year-old boy has NHL on MTX, Adriamycin, Oncovin, Endoxan \& prednisolone (IV), he started to complain of severe persistent headache, MRI was done 2 days after his complain, it shows: a)FLAIR WI: shows areas of high SI in parieto-occipital regions (more evident on the left side). b) DWI: shows no areas of diffusion restriction. c) T1WI: shows areas of low SI in parieto-occipital regions (more evident on the left side) as well as loss of the signal void of the SSS (high SI within). d) T1WI+c: Filling defect within the SSS, suggesting partial thrombosis. Diagnosis: MRI finding consistent with PRES \& partial thrombosis of the SSS.
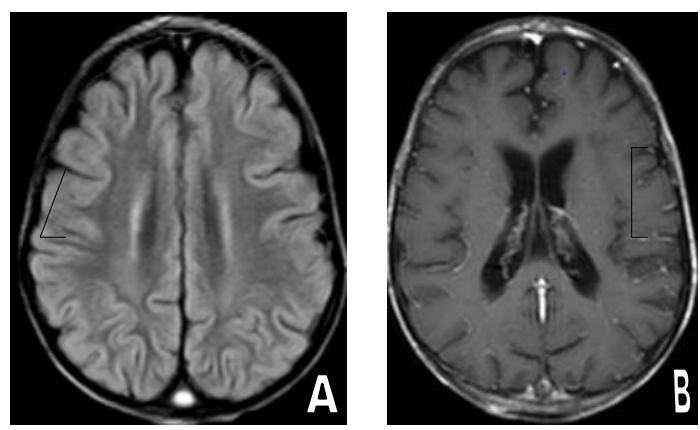

Fig. 14.2 Total resolution of the previously noted abnormal signal intensity within the parieto-occipital regions as seen in the a)FLAIR image, yet further progression of the previously noted SSS thrombosis in which there is more filling defect in the b) post contrast study.
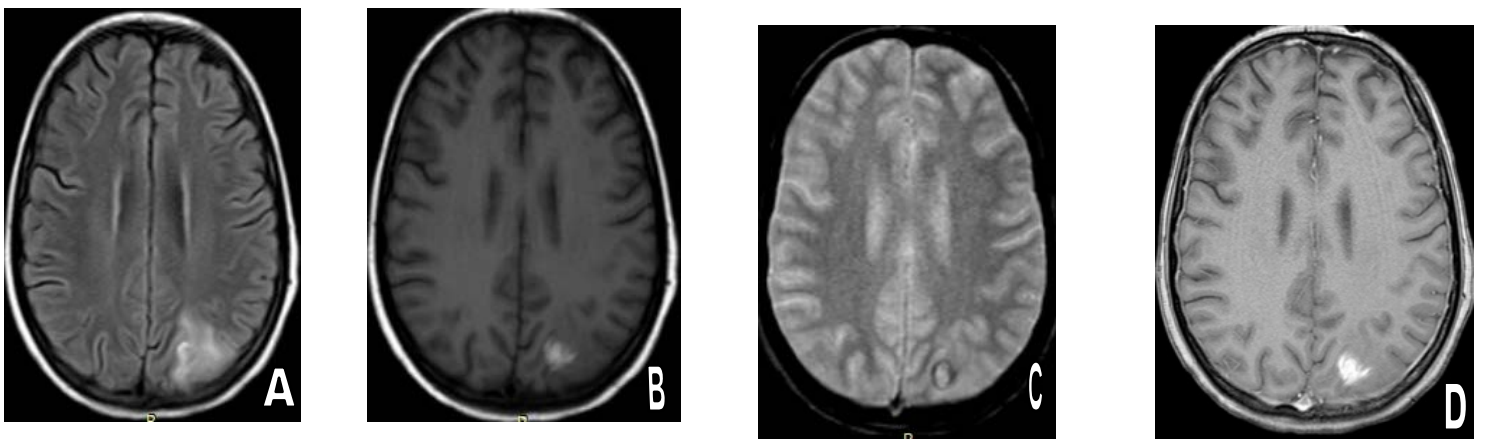

Fig 15.1 13-year-old girl has ALL on high dose MTX (IV), Ca-leucoverin\& 6-mercapto-purine (orally). She started to complain of severe persistent headache, MRI was done 2 days after her complain, it shows : a)FLAIR: Areas of high SI seen at the left parieto- occipital regions. b) TIWI :The lesions are not identified unless foci of high SI detected on the left parieto-occipital region denoting hemorrhage. c) T2*WI showing blooming artifact on the afro-mentioned region, denoting Hge. d) No evidence of contrast enhancement in the post contrast study .Diagnosis: MRI finding consistent of atypical PRES. 


\section{Samar Ramzy Ragheb ${ }^{*}$ and Sherine Mohamed Sharara}
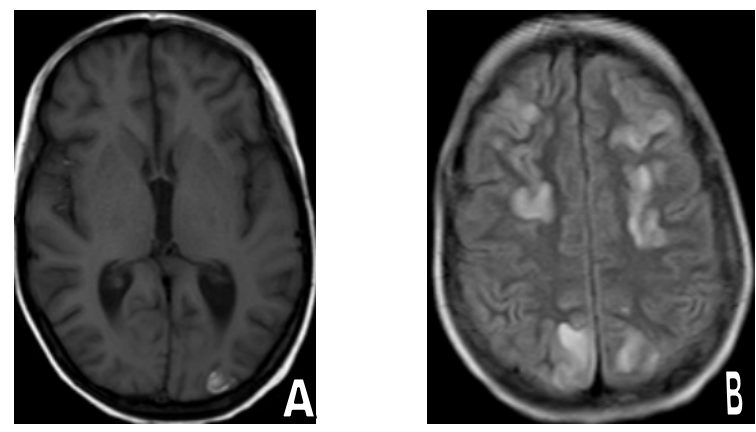

Fig. 15.2 (a): FLAIR: Progression of the previously noted abnormal SI with newly developed bilateral rather symmetrical areas of high signal seen at the subcortical WM of the parietal \& occipital regions. a)T1WI: Still noted foci of high SI denoting persistence of the hemorrhagic component.

\section{Relation between Grading and sequel of PRES:}

The patients with PRES were divided according to their sequel into 2 groups: Group A with complete or near complete resolution of the radiological and neurological manifestations of PRES and group B with stationary course of the manifestations or patients are in coma or died.

13 out of 16 patients that underwent follow up MRI show complete resolution \& near complete resolution that represents $81.25 \%$ \& are classified as group A. 3 out of 16 patients $(18.75 \%)$ show stationary course $\&$ progressive course $\&$ are classified as group B

3 out of 16 patients that underwent follow up MRI which are classified as group B have severe, extensive, and confluent involvement of 3 or all lobes, or involvement of the following: $\mathrm{CC}, \mathrm{BG}$ thalami, or internal capsules, or extensive involvement of the posterior fossa (Grade 4); all of them died or had stationary course.

13 out of 16 patients that underwent follow up MRI which are classified as group A showing minimal (Grade 1), mild (Grade 2) and moderate (Grade 3) grade of involvement; $81.25 \%$ showed complete or near complete resolution (Group A).

This study showed that the sequelae of patients with grade 4 was significantly worse prognosis than the sequel of patients with grade 1, 2 and 3, although no significant difference was found between the first three grades.

Relation between diffusion restriction, hemorrhage \& enhancement to Sequelae of PRES:

In current study $(45.16 \%, 14$ cases out of 31) patients showed restricted diffusion indicating progression to ischemic infarction. Only 8 patients of them underwent follow up MRI, all of them showed regressive course of the disease. Regarding the 3 cases of contrast enhancement, only one case underwent follow up MRI \& shows regressive course of the disease. The only one case of hemorrhage showed regressive course.

\section{DISCUSSION}

CNS toxicity is one of the commonest finding of chemotherapeutic agents. This toxicity can be manifested in many ways, including encephalopathy syndrome, fits, headache, cerebrovascular sequel, loss of vision, cerebellar dysfunction, and damage of spinal cord (myelopathy). For many agents, such toxicity depends on the route of administration and cumulative dose.

In current study we investigated fifty cases of different cancers with CNS radiological findings and had received chemotherapeutics according to their 
primary cancer protocol. PRES was found to be the most common complication (62\%) followed by Cerebral venous thrombosis (CVT) (32\%). PRES and CVT typically presents with signs and symptoms of increased ICP (nausea, vomiting, headache and blurred vision), lethargy, seizures, disorientation and speech impairment.

In current study, the commonest presenting symptom was headache followed by seizures that was in agreement with Alvis et al. ${ }^{[11]}$, who has stated that headache is the most common presentation. It was present in more than $85 \%$ of patients. In contrast Hobson et al. ${ }^{[12-14]}$ found that the most common presentations are seizures, headache, loss of vision, and altered mental status.

In cases of PRES; MR imaging is the favored modality of investigation for early diagnosis of PRES, in many situations, CT imaging can be normal and therefore should not provide reassurance ${ }^{[15]}$.

In current study, the commonest involved typical locations were the parietooccipital lobes $(90.3 \%, 28$ cases out of 31 ,). However the most atypical location affected is the cerebellum $(22.58 \%, 7$ cases out of $31)$, followed by frontal lobe $(19.35 \%, 6$ cases out of 31$)$, then temporal lobe $(9.6 \%, 3$ cases out of 31). Basal ganglia, brain stem \& corpus callosum were the least to be affected (3.2\%, only one case out of 31 for each).

That is in agreement with Raman et al. ${ }^{[16]}$ that reported that the commonest involved typical locations were the parietooccipital lobes $(n=92 ; 100 \%)$. However, other atypical regions involved were the frontal lobes $(30 \%)$, basal ganglia $(22 \%)$, cerebellum $(17.4 \%)$, brainstem $(9 \%)$, temporal lobes $(8.6 \%)$ and thalamus $(4 \%)$.

In our patients we found that the cerebral cortex was the predominant involvement of the PRES lesions, yet it may appear predominantly subcortical or involving both of them.
The white matter abnormal signal intensity of PRES may be unilateral or bilateral. Current study showed that bilaterality of the lesions was more common. However, it was more common to be asymmetrical.

This study showed that typical PRES represent $(32.26 \%)$ of cases, yet the atypical PRES represent $(67.74 \%)$ being classified as atypical presentation and atypical location.

In current study, the pattern of typical PRES identified was bilateral \& symmetrical white-matter abnormal signals in vascular watershed areas in the posterior regions of cerebral hemispheres, affecting mainly the parietal and occipital lobes.

The atypical PRES was considered if there was involvement of any area of the brain other than the parieto-occipital regions, lesion asymmetry, contrast enhancement, restricted diffusion or hemorrhage.

This study showed that 14 out of 31 cases with atypical location, classified as follow: $(50 \%, 7$ cases out of 14) had cerebellar involvement, $(42.8 \%, 6$ cases out of 14$)$ the frontal lobe was involved, $(21.4 \%$, 3 cases out of 14) the temporal lobe was involved. Other atypical locations present in our study include brain stem, basal ganglia $\&$ corpus callosum, in which only 1 case (7.1\% each)present in each place.

In current study 21 out of 31 cases were described as atypical PRES. The commonest atypical presentation reported was asymmetrical distribution of the lesion $(85.7 \%, 18$ cases out of 21$)$, followed by restricted diffusion $(66.6 \%, 14$ cases out of $21)$, then contrast enhancement $(14.2 \%, 3$ cases out of 21$)$ and hemorrhage ( $4.7 \%$, only one case out of 21).

Our results showed that the sequel of patients with grade 4 (involving the brain stem, basal ganglia and thalami) have significantly worse prognosis than the sequel of patients with grade 1,2 and 3, although no significant difference was found between 


\section{Samar Ramzy Ragheb ${ }^{*}$ and Sherine Mohamed Sharara}

the first three grades, so we found significant relation between grading of PRES distribution and outcome of patients.

\section{Conclusion}

So Diffusion weighted imaging (DWI) should be utilized for early diagnosis and follow- up of patients with suspected PRES in order to prevent progressive and irreversible neurological deterioration with cerebral ischemia and infarction.

\section{REFERENCES}

1. Wang TY, Yen HJ, Hung GY., et al. (2011): "A rare complication in a child undergoing chemotherapy for acute lymphoblastic leukemia: superior sagittal sinus thrombosis" Journal of the Chinese Medical Association, vol. 74, no. 4, pp. 183-187.

2. H. Cohen, B. Bielorai, D. Harats, A. Toren, O. Pinhas-Hamiel (2010): " Conservative treatment of L-asparaginase-associated lipid abnormalities in children with acute lymphoblastic leukemia" Pediatr Blood Cancer, 54 , pp. 703-706.

3. Sioka C \& Kyritsis AP (2009): Central and peripheral nervous system toxicity of common chemotherapeutic agents. Cancer Chemother Pharmacol; 63:761-7.

4. Argyriou AA, Koltzenburg M, Polychronopoulos P, Papapetropoulos S, Kalofonos HP (2008): "Peripheral nerve damage associated with administration of taxanes in patients with cancer". Crit Rev Oncol Hematol 66:218-228.

5. Richards A, Marshall H \&McQuary A (2010): Evaluation of methylene blue, thiamine, and/or albumin in the prevention of ifosfamide-related neurotoxicity. J Oncol Pharm Pract.17(4):372-80.

6. Fugate JE, Claassen DO, Cloft HJ., et-al. (2010): Posterior reversible encephalopathy syndrome: associated clinical and radiologic findings. Mayo Clin. Proc. 85 (5): 427-32.
7. Bhojwani D, Sabin ND, Pei D, et al., (2014): Methotrexate-induced neurotoxicity and leukoencephalopathy in childhood acute lymphoblastic leukemia. J Clin. Oncol; 32 (9):949-59.

8. Reddick WE, Conklin HM.(2010): "Impact of acute lymphoblastic leukemia therapy on attention and working memory in children". Expert Rev Hematol.;3:655-659.

9. Vagace JM, Caceres-Marzal C, Jimenez M, et al. (2011): "Methotrexate-induced subacute neurotoxicity in a child with acute lymphoblastic leukemia carrying genetic polymorphisms related to folate homeostasis". Am J Hematol.;86:98-101.

10. Lu CX, Du Y, Xu XX et-al. (2012): Multiple occipital defects caused by arachnoid granulations: Emphasis on T2 mapping. World J Radiol. 4 (7): 341-4.

11. Alvis-Miranda HR, Milena CastellarLeones S, Alcala-Cerra G, et al., (2013): Cerebral venoussinus thrombosis. J Neurosci Rural Pract. (4):427-38.

12. Hobson EV, Craven I \& Blank SC. (2012):Posterior reversible encephalopathy syndrome: a truly treatable neurologic illness. Perit Dial Int.; 32(6):590-4.

13. Bartynski W (2008):"Posterior reversible encephalopathy syndrome, part 1: fundamental imaging and clinical features". AJNR Am J Neuroradiol; 29:1036-42.

14. Bavikatte $\mathrm{G}$, Gaber $\mathrm{T}$, Eshiett MUet al. (2010): "Posterior reversible encephalopathy syndrome as a complication of Guillain-Barré syndrome”. J Clin Neurosci; 17:924-6.

15. Roth C \&Ferbert A. (2011): The posterior reversible encephalopathy syndrome: what's certain, what's new? Pract Neurol 11:136-44.

16. Raman R, Devaramane R, Jagadish GM, et al. (2017): Various Imaging Manifestations of Posterior Reversible Encephalopathy Syndrome (PRES) on Magnetic Resonance Imaging (MRI). Pol J Radiol. 82:64-70. 


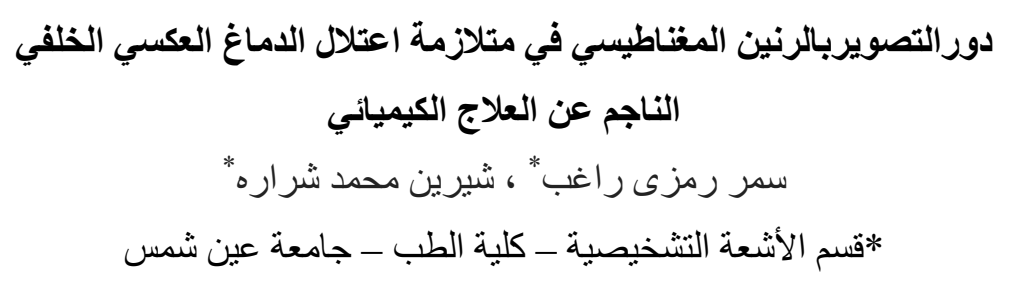

المقدمة: متلازمة اعتلال الدماغ العكسي الخلفي هي أحد الآثار الجانبية السمية العصبية الثائعة للعلاجا لكيميائي. الهدف من البحث: إظهار أهمية التصوير بالرنين المغناطيسي في الكثف المبكر عن السمية العصبية التي يسببها العلاج الكيمبائي.

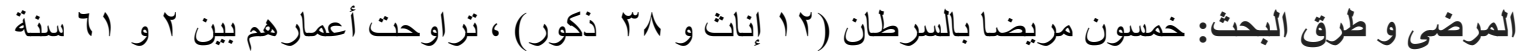
على العلاج الكيميائي الذي له له مظاهر عصبية. تم إجراء التصوير بالرنين المغناطيسي لتقييم النتائج الإشعاعية للمظاهر العصبية.

النتائج: تم العثور على اس حالة لديها متلازمة اعتلال الدماغ العكسي الخلفي، في حين أظهرت الحالات الأخرى الـ 19 19 الخلاصة: أظهر التصوير بالرنين المغناطيسي دورًا مهمًا في الكثف عن متلازمة اعتلال الدماغ العكسي الخلفي في مرضى السرطان بسبب العلاج الكيميائي. 